\title{
A new simple method of staining exogenous surfactant in experimental research
}

\author{
M.F. Krause*, M. Orlowska-Volk**, E.R. Hendrik", D. Gommers", B. Lachmann ${ }^{\#}$
}

\begin{abstract}
A new simple method of staining exogenous surfactant in experimental research. M.F. Krause, M. Orlowska-Volk, E.R. Hendrik, D. Gommers, B. Lachmann. (C)ERS Journals Ltd 2000.

ABSTRACT: Two commonly used techniques in experimental lung research have helped to determine which variables influence surfactant distribution within the lung: radioactive labelling of surfactant components and admixture of coloured microspheres to surfactant. However, neither technique allows the description of surfactant distribution at the alveolar level. The aim of this study was to establish a new technique using histology colourants for admixture to exogenous surfactant to make exogenous surfactant visible by light microscopy.

In a step by step approach the authors evaluated the properties of a variety of green colourants when added to a natural porcine surfactant preparation for their ability to homogeneously mix with surfactant, to bind to surfactant, to adhere to a glass slide, to not be "overstained" by standard haematoxylin-eosin and Elastica van Giesson staining, to not influence in vitro surface tension properties of surfactant using a Wilhelmy balance, to not influence oxygenation and ventilation in a lung-lavage rat model and to preserve their colour and adherence to exogenous surfactant on lung specimens visualized by light microscopy.

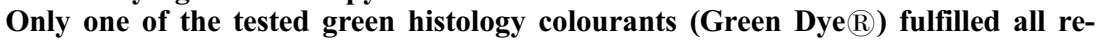
quirements and showed a brilliant green colour in a distribution pattern typical of surfactant at the alveolar level.

It is concluded that the authors have established a new, simple and inexpensive method of staining exogenous surfactant for evaluation of its distribution by light microscopy at the alveolar level.

Eur Respir J 2000; 15: 949-954.
\end{abstract}

\begin{abstract}
*Dept of Pediatrics, and **Dept of Pathology, Albert-Ludwigs-University, University Hospital, Freiburg, Germany, \#Dept of Anesthesiology, Erasmus University, Rotterdam, the Netherlands.
\end{abstract}

Correspondence: M.F. Krause, University Children's Hospital, Mathildenstrasse 1, D-79106, Freiburg, Germany. Fax: 49 7612704399

Keywords: Alveoli exogenous surfactant histology colourants light microscopy stained surfactant surfactant distribution

Received: July 201999

Accepted after revision December 101999

Sources of financial support (for M.F. Krause): Beringer-Stiftung, Freiburg, Germany; Wissenschaftliche Gesellschaft, Freiburg, Germany; Departmental fund, Department of Anesthesiology, Erasmus University, Rotterdam, The Netherlands.
Surfactant replacement therapy for neonatal respiratory distress syndrome has now been used as a standard therapeutic modality since before 1989 [1]. Several variables determine surfactant distribution after its instillation into the endotracheal tube, such as the surfactant preparation itself, age of the newborn at treatment, volume and concentration of surfactant, the time interval for surfactant administration if repeatedly used, alternative ways of surfactant administration (e.g. nebulization), and the style of mechanical ventilation [2]. Homogeneous distribution of exogenous surfactant is of paramount importance for a favourable clinical response in treated infants because atelectatic lung areas with a substantial intrapulmonary right-to-left shunt and consecutive hypoxia should be avoided. However, most data focusing on surfactant distribution come from experimental research using different species of animals allowing histological or other processing of the lungs at the end of the experiment.

Two commonly used techniques have shed some light on surfactant distribution in experimental research: radioactive labelling of dipalmitoyl phosphatidylcholine or other surfactant components, and admixture of coloured microspheres to surfactant. Determination of the distribution of radioactively labelled surfactant requires considerable effort such as collaboration with nuclear medicine departments $[3,4]$ and expensive discarding of these radioactively polluted animals. Lung processing for evaluating distribution of exogenous surfactant using the coloured microsphere technique needs additional staff and an extra session to cut the lung into 50-70 pieces before washing out the colorant affixed to the microspheres $[5,6]$.

Therefore, the aim of this study was to establish a new method for experimental lung research which allows the description of surfactant distribution at the alveolar level when analysing histological specimens. The authors studied eight histological colourants for admixture to exogenous surfactant in a step-by-step approach to visualize exogenous surfactant when using light microscopy for evaluation of lung specimens.

\section{Material and methods}

\section{Colourants}

A variety of green colourants was tested to provide a powerful optical contrast because the standard staining techniques with haematoxylin-eosin (HE) or Elastica van Giesson (EvG) for the histological evaluation of lung 
specimens stain structures predominantly a deep blue, red and yellow.

The following substance was tested for its ability to homogeneously mix with surfactant, to adhere to a glass slide, and to preserve its original green colour on a slide when mixed with surfactant and stained with either HE or EvG: Green Tissue Marking Dye $\mathbb{R}$ (Green Dye: WAKChemie Medical GmbH, Bad Soden, Germany).

Green Dye was diluted 1:10 with normal saline. The diluted colourant was then further diluted by hand stirring one part with nine parts of surfactant until total homogeneous mixing was achieved, leaving a final concentration of $1 \%$ of the original dye. After the drying process the slide was stained with $\mathrm{HE}$ and EvG and checked for adherence of the colourant to the slide and maintenance of its original green colour.

\section{In vitro testing of stained surfactant}

The authors used a freeze dried natural surfactant preparation isolated from porcine lungs as previously described [7]. It consists of $\sim 90-95 \%$ phospholipids, $1 \%$ hydrophobic proteins (surfactant proteins $\mathrm{B}$ and $\mathrm{C}$ ) and $1 \%$ free fatty acids, the remainder being other lipids such as cholesterol and glyceride. There is no surfactant protein A in this preparation. Surfactant powder was added to warmed normal saline and stirred to a final concentration of 40 $\mathrm{mg} \cdot \mathrm{mL}^{-1}$.

Stained and uncoloured surfactant preparations were tested for their minimal surface tensions using a modified Wilhelmy balance (E. Biegler, Mauerbach, Austria) by applying increasing amounts $(100 \mu \mathrm{L}, 200 \mu \mathrm{L}$ and $500 \mu \mathrm{L})$ to the surface of a warmed saline filled trough [8]. The surface area was compressed and expanded with a cycling time of 3 min per cycle and maximum and minimum surface areas of 64 and $12.8 \mathrm{~cm}^{2}$, respectively $(100 \%$ and $20 \%$ ). The minimal surface tension was measured after three cycles at $20 \%$ surface area, and was expressed in millinewtons per metre.

\section{In vivo testing of stained surfactant}

This part of the study was approved by the Institutional Animal Committee at the Erasmus University Rotterdam; care and handling of the animals were in accord with the Helsinki convention.

The physiological studies were performed in male Sprague-Dawley rats $(n=10)$ with a body weight of $\sim 300 \mathrm{~g}$. After the induction of anaesthesia with nitrous oxide, oxygen and enflurane $(66 / 33 / 1-2 \%)$, a polyethylene catheter ( $0.8 \mathrm{~mm}$ outer diameter) was inserted into the carotid artery for drawing arterial blood samples. Before tracheotomy, the animals received pentobarbital sodium, $30 \mathrm{mg} \cdot \mathrm{kg}$ body weight $^{-1}$, i.p. After a metal cannula had been inserted into the trachea it was secured in place by a peritracheal ligature. The animals were then mechanically ventilated with a Servo Ventilator 300 (Siemens-Elema, Solna, Sweden) in parallel (five animals simultaneously) in a pressure control mode with a continuous flow in the tubings. Muscle relaxation was achieved using pancuronium bromide, 0.6 $\mathrm{mg} \cdot \mathrm{kg}$ body weight ${ }^{-1}$, i.m.
The following ventilator settings were used: frequency of 30 breaths per minute, positive end-expiratory pressure (PEEP) of $200 \mathrm{~Pa}$ ( $2 \mathrm{mbar}$ ), inspiratory/expiratory ratio of $1: 2$, inspiratory oxygen fraction $\left(F \mathrm{I}, \mathrm{O}_{2}\right)$ of 1.0 and a peak inspiratory pressure (PIP) of $1.2 \mathrm{kPa}(12 \mathrm{mbar})$ yielding a carbon dioxide tension in arterial blood $\left(\mathrm{Pa}_{\mathrm{a}} \mathrm{CO}_{2}\right)$ of 3.7-5.3 $\mathrm{kPa}(28-40 \mathrm{mmHg})$. Anaesthesia was maintained with hourly injections of $30 \mathrm{mg} \cdot \mathrm{kg}$ body weight ${ }^{-1}$ pentobarbital i.p. and $0.6 \mathrm{mg} \cdot \mathrm{kg}$ body weight ${ }^{-1}$ pancuronium i.m. Body temperature was kept within the normal range by means of a heating pad.

Respiratory failure was induced by repeated bronchoalveolar lavage as described by LACHMANN et al. [9]. Immediately before the first lavage PIP and PEEP were increased to pressures of 2.6/0.6 $\mathrm{kPa}$ (26/6 mbar). Each lavage involved the instillation and removal of $30 \mathrm{~mL} \cdot \mathrm{kg}$ body weight ${ }^{-1}$ of warmed saline via the tracheal cannula carried out over a 30 -s period. Airway lavage was repeated every $5 \mathrm{~min}$ until oxygen tension in arterial blood $\left(P \mathrm{a}, \mathrm{O}_{2}\right)$ was $<10.6 \mathrm{kPa}(80 \mathrm{mmHg})$. The animals were then randomly divided into two groups of five animals each: the first group received $1 \mathrm{~mL}$ of stained surfactant with Green Dye, and the second group served as a control group receiving $1 \mathrm{~mL}$ of uncoloured surfactant at a concentration of $40 \mathrm{mg} \cdot \mathrm{mL}^{-1}$.

Additional measurements of blood gases $\left(\mathrm{Pa}, \mathrm{O}_{2}\right.$ and $\left.P \mathrm{a}, \mathrm{CO}_{2}\right)$ were made at 5, 30, 60, 90 and $120 \mathrm{~min}$ after surfactant instillation. All ventilator settings were held constant for this observational period of $2 \mathrm{~h}$ and were changed thereafter by steps of $200 \mathrm{~Pa}(2 \mathrm{mbar})$ to $2.4 / 0.4$ $\mathrm{kPa}$ (24/4 mbar) (PIP/PEEP), 2.2/0.2 kPa (22/2 mbar), 2.0/ $0.0 \mathrm{kPa}(20 / 0$ mbar) and again $2.6 / 0.6 \mathrm{kPa}$ (26/6 mbar) every $10 \mathrm{~min}$ to test deflation stability of the lungs.

At the end of each experiment the animals were killed with an overdose of pentobarbital, and the lungs were removed from the chest for histological processing.

\section{Histologic processing}

After tying off the trachea to prevent washout of stained surfactant the lungs were put en bloc with the heart and thymus into $4 \%$ formaldehyde for several days before the histological processing was carried out.

After embedding the specimens in paraffin four cuts of each lung at a width of $4 \mu \mathrm{m}$ were taken from central right, central left, peripheral right and peripheral left locations and put on slides for fixation. All slides were stained with $\mathrm{HE}$ and EvG using standard methods. Photographs were taken to demonstrate the pattern of surfactant distribution within the lungs.

\section{Chemistry of Green Dye and binding to surfactant}

According to information from the manufacturer (WAKChemie Medical GmbH) Green Dye consists of green colourants fixed to latex microparticles which are inert to physiological fluids.

To assess binding of Green Dye to surfactant in vitro the following centrifugation protocol was used: three tubes were prepared containing: 1) Green Dye diluted 1:10 with normal saline and mixed with an equal amount of $50 \%$ dextrose in sterile water; 2) surfactant without admixture of 
Green Dye with 50\% dextrose; and 3) surfactant with admixture of Green Dye and 50\% dextrose. The three tubes were then placed in an ultracentrifuge (Model L7-55; Beckman Instruments, Palo Alto, CA, USA) at $40,000 \times g$ and $4^{\circ} \mathrm{C}$ for $20 \mathrm{~min}$ under vacuum conditions.

\section{Statistical methods}

All data are expressed as mean \pm SD. A Wilcoxon rank sum test was used to calculate differences between the two groups. Repeated measures analysis of variance was used to assess differences in blood gases in change over time. An unpaired t-test [10] was used to determine whether there were differences between intervention and control group at individual observational times. All analyses were performed using the software program GraphPad Prism 2.0 (GraphPad Software, San Diego, CA, USA).

Statistical significance was accepted at $\mathrm{p}<0.05$.

\section{Results}

\section{Colourants}

By light microscopy Green Dye showed a stable fixation to the glass slide and a brilliant green colour when mixed with surfactant and "overstained" with HE and EvG.

Other colourants were also tested, but tests were abandoned at different stages because the requirements were

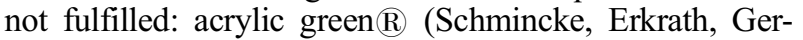
many); methyl green $\mathbb{R}$; light green $\mathrm{SF} \mathbb{R}$; Janus green

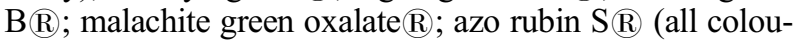
rants: Chroma, Köngen, Germany); resolin brillant blue BGLN 200\%®; resolin brillant yellow $10 \mathrm{GN} 400 \% \AA$ (DyStar, Leverkusen, Germany).

In vitro testing of stained surfactant with a Wilhelmy balance

Two sets of measurements were performed (table 1): one set with increasing amounts of surfactant immediately after preparation, and another set $3 \mathrm{~h}$ after preparation reflecting the time period used for the in vivo testing of stained surfactant in rats yielding no differences bet-

Table 1. - Minimal/maximal surface tensions with and without stained surfactant (surf.) preparations

\begin{tabular}{lcc}
\hline & \multicolumn{2}{c}{ Minimal/maximal surface tension $\mathrm{mN} \cdot \mathrm{m}^{-1}$} \\
\cline { 2 - 3 } & $\begin{array}{c}\text { Immediately after } \\
\text { preparation }\end{array}$ & $\begin{array}{c}\text { After } 3 \mathrm{~h} \text { of } \\
\text { incubation }\end{array}$ \\
\hline Surf. + Green Dye & $16.5 / 25.9$ & $15.5 / 29.3$ \\
$100 \mu \mathrm{L}$ & $15.6 / 26.2$ & $14.3 / 25.3$ \\
$200 \mu \mathrm{L}$ & $8.0 / 16.1$ & $11.7 / 17.3$ \\
$500 \mu \mathrm{L}$ & $16.8 / 30.0$ & $15.9 / 26.8$ \\
Control: uncoloured surf. & $14.0 / 26.5$ & $14.5 / 26.5$ \\
$100 \mu \mathrm{L}$ & $8.0 / 13.9$ & $10.5 / 17.0$ \\
$200 \mu \mathrm{L}$ & $500 \mu \mathrm{L}$ & \\
\hline
\end{tabular}

A surfactant concentration of $40 \mathrm{mg} \cdot \mathrm{mL}^{-1}$ was used and added to the surface of a warmed saline-filled trough of a modified Wilhelmy balance. There were no statistically significant differences between groups. ween the stained and the uncoloured surfactant preparations.

pH-measurement for Green Dye yielded a value of 6.5. When mixed with surfactant (one part colour and nine parts surfactant), the values rose to 7.0.

Stained surfactant was incubated in a cuvette at room temperature for 3 days; however, no sedimentation or dissolution of the different components could be discerned. The same procedure was undertaken adding $4 \%$ formaldehyde with 1 part of formaldehyde and one part of coloured surfactant. Again, there were no signs of sedimentation or dissolution.

In vivo testing of stained surfactant in a lung-lavage rat model

There were no differences between groups with regard to body weight (Green Dye: $290 \pm 11 \mathrm{~g}$; control: $301 \pm 17 \mathrm{~g}$, respectively) and the number of lavages applied (Green Dye: $6.0 \pm 0.7$; control: $5.8 \pm 0.8$, respectively).

Figure 1 shows all values for $\mathrm{Pa}, \mathrm{O}_{2}$ and $\mathrm{Pa}_{\mathrm{a}} \mathrm{CO}_{2}$ before and after lavage, and for the 2-hour observation period following surfactant instillation and the three-step reduction of ventilator pressures to assess deflation stability of the terminal airways, yielding no statistically significant differences between the two groups.
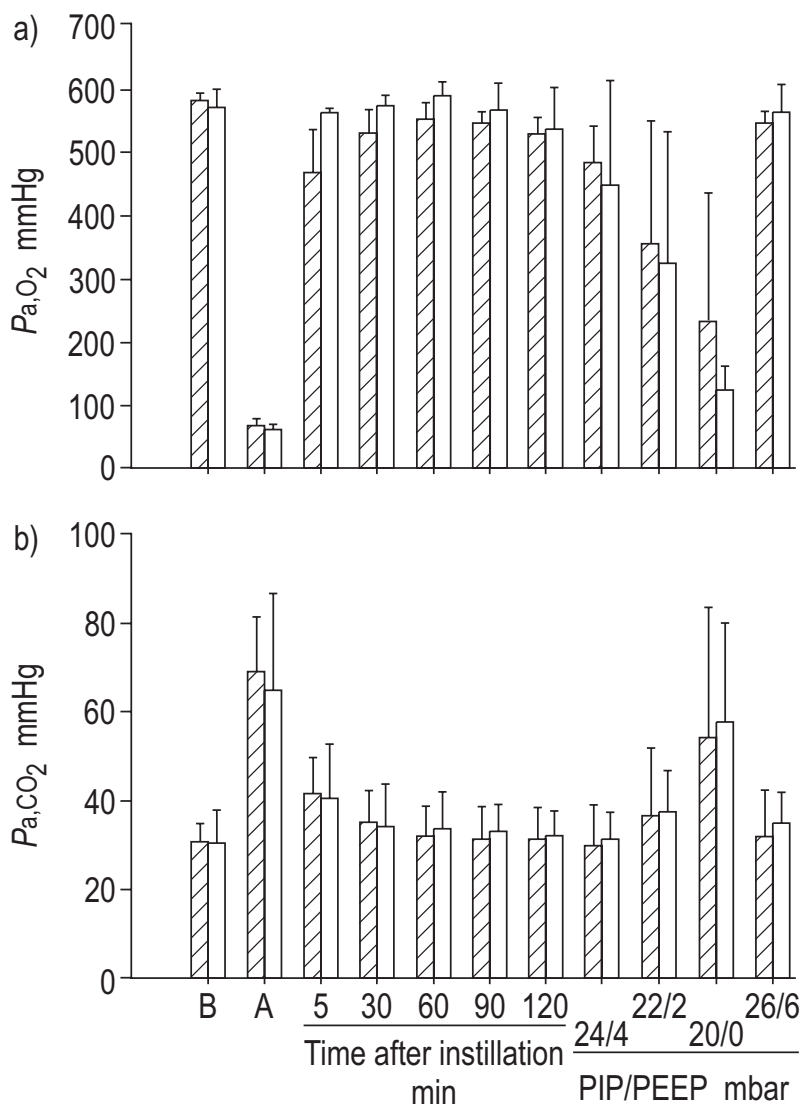

Fig. 1. - Data on oxygenation and ventilation of the two groups: a) arterial oxygen tension $\left(\mathrm{Pa}_{\mathrm{a}} \mathrm{O}_{2}\right)$; and $\left.\mathrm{b}\right)$ arterial carbon dioxide tension $\left(P \mathrm{a}_{2} \mathrm{CO}_{2}\right)$. Data are presented as mean $\pm \mathrm{SD}(\mathrm{n}=5)$. There were no significant differences between Green Tissue Marking Dye $\mathbb{B}(\mathbb{Z})$ and uncoloured surfactant (control; $\square$ ). B: before lavage; A: after lavage. 1 $\mathrm{mmHg}=0.133 \mathrm{kPa}$ 


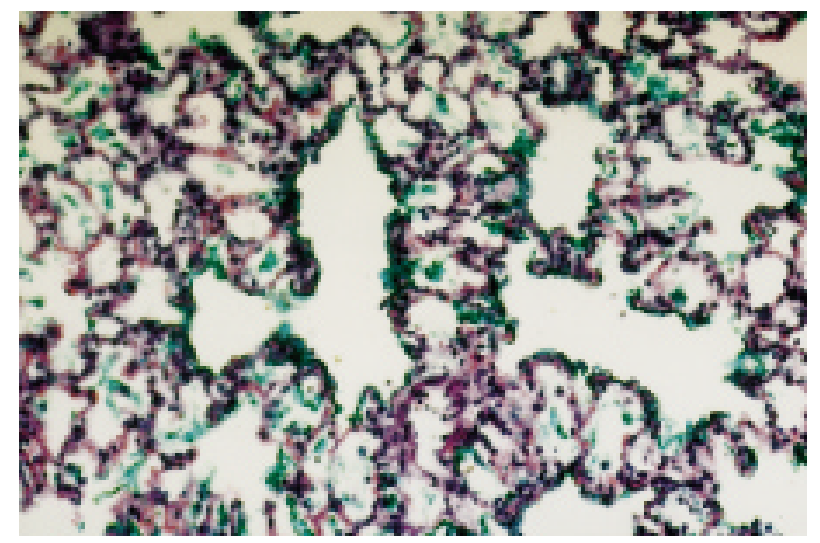

Fig. 2. - Homogeneous distribution of green coloured surfactant in the alveoli and along the alveolar septa. Haematoxylin-eosin stain; internal scale bar $=25 \mu \mathrm{m}$.

\section{Histological processing}

Surfactant coloured with Green Dye could easily be found on all slides using both staining techniques. Figure 2 (HE staining) shows homogeneous depositions of green coloured surfactant in the alveoli and along the alveolar septa, whereas almost no green material could be found in the larger bronchi indicating good spreading properties of this coloured surfactant preparation. Figure 3 shows a layer of stained surfactant adjacent to the alveolar epithelium. Hyaline membranes could be identified but always with very little incorporation of stained surfactant (fig. 4). The same frequency of hyaline membranes was present on the control group slides. Macrophages within the alveoli showed incorporation of exogenous surfactant. As usual in this lung lavage model, the interstitial lung tissue was irregularly invaded by granulocytes also incorporating surfactant. Figure 5 shows the incorporation of coloured surfactant by alveolar epithelial cells, most likely type II pneumocytes, which are known to recycle and store endogenous and exogenous surfactant from the alveolar layer [11]. Green coloured surfactant was also preserved on slides with EvG staining (fig. 6).

\section{Binding of Green Dye to surfactant}

When incubated with equal amounts of 50\% dextrose and rotated at $40,000 \times g$, Green Dye alone sunk to the

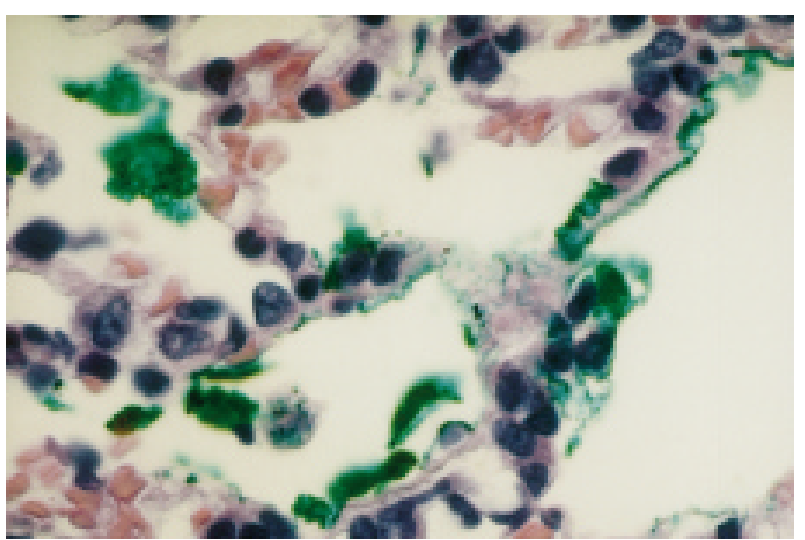

Fig. 3. - Layer of coloured surfactant adjacent to the alveolar epithelium. Haematoxylin-eosin, oil; internal scale bar $=10 \mu \mathrm{m}$.

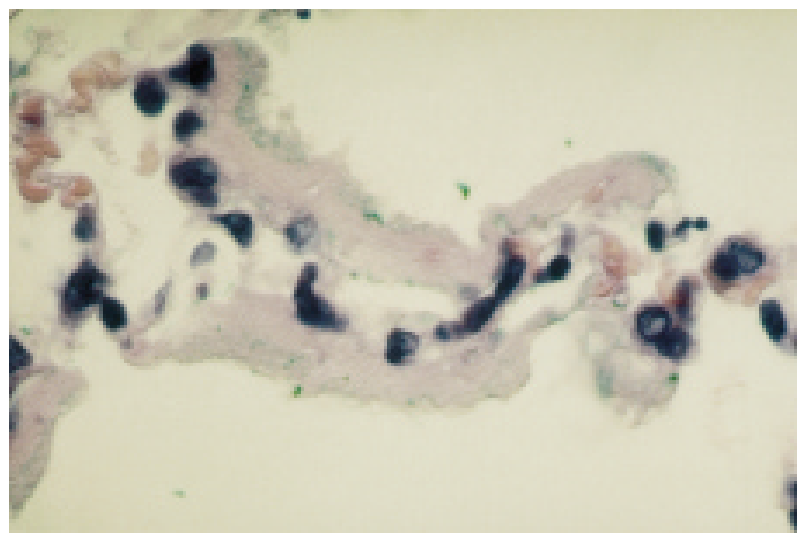

Fig. 4. - Hyaline membranes, with almost no incorporation of stained surfactant. Haematoxylin-eosin, oil; internal scale bar $=10 \mu \mathrm{m}$.

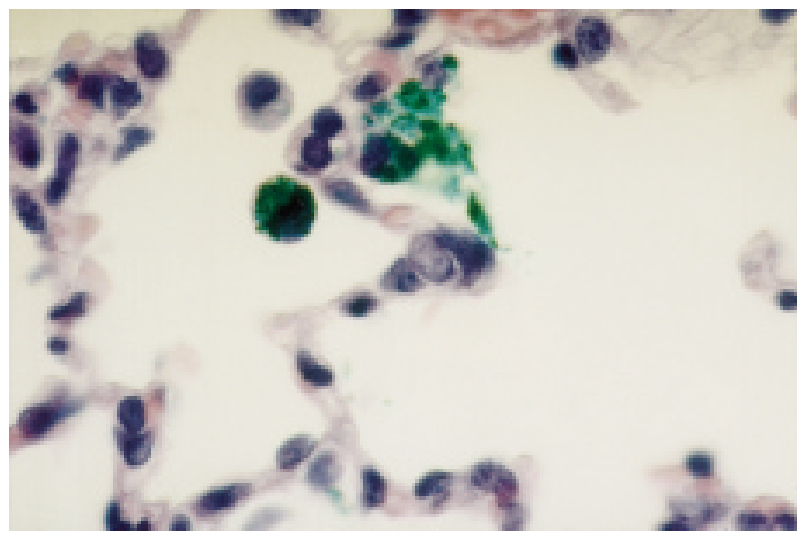

Fig. 5. - Ingestion of coloured surfactant by type II pneumocytes and macrophages. Haematoxylin-eosin, oil; internal scale bar $=10 \mu \mathrm{m}$.

bottom of the tube. In contrast uncoloured surfactant appeared as a supernatant floating on the liquid surface as did stained surfactant leaving small amounts of unbound Green Dye on the bottom. It is concluded that Green Dye is indeed chemically bound to the bulk of surfactant without influencing the surface tension properties of exogenous surfactant.

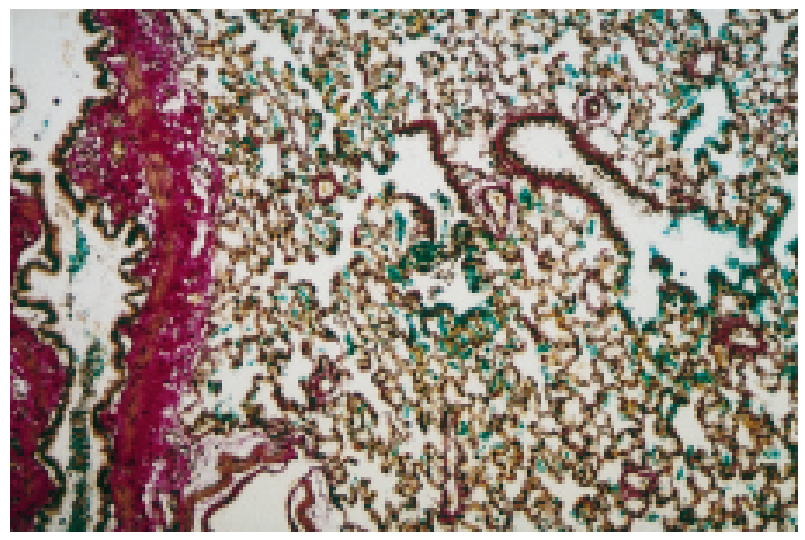

Fig. 6. - Distribution of coloured surfactant. Elastica van Giesson; internal scale bar $=40 \mu \mathrm{m}$. 


\section{Discussion}

The authors succeeded in identifying by trial and error a colourant originally designed for histology use which easily dissolved with a porcine natural surfactant preparation without disturbing the surfactant's in vitro or in vivo surface tension properties. Moreover, it could be easily detected on histology slides of rats' lungs still exerting its original brilliant green colour when staining the lung specimens with HE or EvG. On histological examination the coloured surfactant was only very slightly incorporated into hyaline membranes but was present in macrophages, granulocytes and type II pneumocytes. It did not cross alveolar membranes or pulmonary capillary endothelium (when, postmortem, the coloured surfactant was injected into the right ventricle and circulated through the pulmonary blood vessels). No toxic effects could be discerned during the in vivo test lasting $\sim 2.5 \mathrm{~h}$, a finding in line with the authors' observation that the green colour was readily bound to surfactant and did not show any signs of sedimentation or dissolution. The manufacturer has stated that no toxic chemicals are mixed to the latex microparticles. It is assumed that chemical binding of the green colorant to surfactant, as demonstrated by ultracentrifugation, is due to binding to the bulk of surfactant components (i.e. phospholipids) in the surfactant preparation used in the present study.

The authors are not aware of any other publication using histology colourants for staining exogenous surfactant in experimental research. However, different techniques have been used in the past to investigate the distribution of exogenous surfactant when instilled into the surfactantdeficient lungs of research animals. Most experimental studies of surfactant distribution have used radiolabelled surfactant or other easily measured substances mixed with surfactant components [11]. JoBE et al. [12] used a trace amount of $\left[{ }^{3} \mathrm{H}\right]$-palmitic acid labelled sheep surfactant to assess its distribution, Gilliard et al. [13] and HELDT et al. $[14]\left[{ }^{3} \mathrm{H}\right]$ choline labelled dipalmitoyl phosphatidylcholine (DPPC), and DAVIS et al. [3] $\left.{ }^{99 \mathrm{~m}} \mathrm{Tc}\right]-$ labelled calf lung surfactant extract. Two differently labelled surfactant preparations $\left(\left[{ }^{3} \mathrm{H}\right]\right.$-choline labelled DPPC and $\left[{ }^{14} \mathrm{H}\right]$-palmitate labelled DPPC) were used by UEDA et al. [15] for differential analysis of various surfactant administration protocols. $\left[{ }^{125} \mathrm{I}\right]$-iodophenyldiazirine bound to albumin was used in the experiment of HENRY et al. [16]. Each technique has different strengths and weaknesses, giving only partial information about surfactant distribution [11]. External scanning has low resolution because of the superposition of the entire thickness of the lungs. This problem may be overcome when the lungs are divided into $\sim 200$ pieces and distribution per piece was measured using $\left[{ }^{141} \mathrm{Ce}\right]$-labelled microspheres mixed with exogenous surfactant [4]. Two different radioisotopes $\left(\left[{ }^{141} \mathrm{Ce}\right.\right.$ and ${ }^{103} \mathrm{Rn}$ ]-labelled microspheres) were used by PLÖTZ et al. [17] to differentiate distribution of a first and a second dose of exogenous surfactant in lung-lavaged rabbits. This technique of dividing lungs into pieces and measuring the amount of surfactant and its marker that distributes with surfactant in each piece has the advantage of being quantitative. However, the lung pieces do not provide information about alveolar distribution or the amount of surfactant not reaching the alveolar level.
Another commonly used technique involves the admixture of coloured microspheres to exogenous surfactant, and the pattern of distribution has been found to be equal to $\left.{ }^{14} \mathrm{C}\right]$-labelled DPPC in rabbit lungs after surfactant depletion [5]. Again, the lungs were cut into 50 [6] to 70 [5] pieces after which the colourant was dissolved from the microspheres and quantified. Thus, it is a very demanding technique which requires additional technical equipment, personnel, and a second session for lung processing alone.

AmirkHANiAn and MerRitT [18] demonstrated that the surface tension properties of natural surfactant preparations tested (including natural surfactants containing surfactant proteins $\mathrm{B}$ and $\mathrm{C}$ ) are preserved within a $\mathrm{pH}$ range of 6.5-7.2, and that the whole range from $\mathrm{pH} 2-8$ is still in accordance with almost unchanged surfactant surface tension properties. Therefore, the $\mathrm{pH}$ of 6.5 of Green Dye is in the ideal range of $\mathrm{pH}$ values for admixture to surfactant preparations.

Some questions remain that cannot be answered with the data from the present study: will the Green Dye/surfactant mixture be stable for $>3 \mathrm{~h}$ after preparation and exert an undiminished physiological activity? Are there toxicological side effects which were not assessed (e.g. through monitoring of cardiovascular parameters)? How much dye is already unbound from surfactant after a study period of 3 $\mathrm{h}$ in vivo and will probably be degraded in a different manner such as unbound excess dye? Is the activity of macrophages or other cells augmented when colourants are added to surfactant? Are there any immunological reactions that were not assessed in this experiment?

In conclusion, the authors have found a stable colourant which allows staining of exogenous surfactant for experimental lung research, when the question of surfactant distribution at the alveolar level is being investigated to optimize e.g. the mode of surfactant administration, timing of surfactant administration or the ventilation strategy. In addition quantitative assessment of surfactant distribution may be optimized by using planimetric systems for counting green stained exogenous surfactant deposits.

Acknowledgements. The authors thank L. Visser-Isles for English language editing.

\section{References}

1. Kattwinkel J. Surfactant - Evolving issues. In: Goldsmith JP, Spitzer AR, eds. Controversies in neonatal pulmonary care. Clin perinatol. Philadelphia, USA, WB Saunders, 1998; pp. 17-32.

2. Jobe AH. Techniques for administering surfactant. In: Robertson B, Taeusch HW, eds. Surfactant therapy for lung disease. New York, USA, Marcel Dekker, 1995; pp. 309-324.

3. Davis JM, Russ GA, Metlay L, Dickerson B, Greenspan BS. Short-term distribution kinetics of intratracheally administered exogenous lung surfactant. Pediatr Res 1992; 31: 445-450.

4. Van der Bleek J, Plotz FB, van Overbeek M, et al. Distribution of exogenous surfactant in rabbits with severe respiratory failure: the effect of volume. Pediatr Res 1993; 34: $154-158$. 
5. Segerer H, van Gelder W, Angenent FWM, et al. Pulmonary distribution and efficacy of exogenous surfactant in lung-lavaged rabbits are influenced by the instillation technique. Pediatr Res 1993; 34: 490-494.

6. Balaraman V, Sood SL, Finn KC, Hashiro G, Uyehara CFT, Easa D. Physiologic response and lung distribution of lavage versus bolus Exosurf in piglets with acute lung injury. Am J Respir Crit Care Med 1996; 153: 1838-1843.

7. Gommers D, Vilstrup C, Bos JAH, et al. Exogenous surfactant therapy increases static lung compliance, and cannot be assessed by measurement of dynamic compliance alone. Crit Care Med 1993; 21: 567-574.

8. Notter RH. Surface chemistry of pulmonary surfactant: the role of individual components. In: Robertson B, Van Golde LMG, Batenburg JJ, eds. Pulmonary surfactant. Amsterdam, The Netherlands, Elsevier, 1984; pp. 17-65.

9. Lachmann B, Robertson B, Vogel J. In vivo lung lavage as an experimental model of respiratory distress syndrome. Acta Anaesthesiol Scand 1980; 24: 231-236.

10. Motulsky H. Intuitive biostatistics. New York, NY, USA, Oxford University Press, 1995; 207-229.

11. Jobe AH. Phospholipid metabolism and turnover. In: Polin RA, Fox WW, eds. Fetal and neonatal physiology. Philadelphia, USA, WB Saunders, 1992; 986-995.

12. Jobe A, Ikegami M, Jacobs H, Jones S. Surfactant and pulmonary blood flow distributions following tratment of premature lambs with natural surfactant. $J$ Clin Invest 1984; 73: 848-856.

13. Gilliard N, Richman PM, Merritt TA, Spragg RG. Effect of volume and dose on the pulmonary distribution of exogenous surfactant administered to normal rabbits or to rabbits with oleic acid lung injury. Am Rev Respir Dis 1990; 141: 743-747.

14. Heldt GP, Merritt TA, Golembeski D, Gilliard N, Bloor C, Spragg R. Distribution of surfactant, lung compliance, and aeration of preterm rabbit lungs after surfactant therapy and conventional and high-frequency oscillatory ventilation. Pediatr Res 1992; 31: 270-275.

15. Ueda T, Ikegami M, Rider ED, Jobe AH. Distribution of surfactant and ventilation in surfactant-treated preterm lambs. J Appl Physiol 1994; 76: 45-55.

16. Henry MD, Rebello CM, Ikegami M, Jobe AH, Langenback EG, Davis JM. Ultrasonic nebulized in comparison with instilled surfactant treatment of preterm lambs. Am J Respir Crit Care Med 1996; 154: 366-375.

17. Plötz FB, Stevens H, Heikamp A, Bambang Oetomo S. Distribution of a second dose of exogenous surfactant in rabbits with severe respiratory failure. Pediatr Res 1995; 37: 476-481.

18. Amirkhanian JD, Merritt TA. The influence of $\mathrm{pH}$ on surface properties of lung surfactants. Lung 1995; 173: $243-254$. 